Book Review

ISSN: 2162-3104 Print/ ISSN: 2166-3750 Online

Volume 6, Issue 1 (2016), pp. 322-324

(C) Journal of International Students

http://jistudents.org/

\title{
Demystifying Outcomes Assessment for International Educators
}

Deardorff, D. (2015). Demystifying outcomes assessment for international educators: A practical approach. Sterling, VR: Stylus Publishing, ISBN: 978-1-62036-128-3 (paperback)

Reviewed by Paige E. Butler, Middlebury Institute of International Studies at Monterey (USA)

Demystifying Outcomes

Assessment for

Internatjona]

Educators

\section{A PRACTICAL APPROACH}

Darla K. Deardorff

Forewords by Trudy W. Banta and Hans de Wit

Darla Deardorff's new book Demystifying Outcomes Assessment for International Educators: A Practical Guide utilizes an applied approach to outcomes assessment in international education. Faculty and practitioners across international education programs and services will find significant value in the materials presented throughout the text.

Concepts and strategies to support the assessment of student learning outcomes in education abroad, international student and scholar services, language program administration, teaching international students, intercultural training programs and other areas of international education will find Deardorff's work useful in practice. The book is constructed to help practitioners at varying career levels and can speak to individuals without assessment experience, as well as provide useful tools and reminders for those who have experience with assessment in international education. Throughout the book, practical tools and strategies that can be utilized throughout the assessment process are discussed and highlighted in an easyto-read format for the busiest of faculty and staff.

The book presents the holistic cycle of assessment with a particular emphasis on assessment design and the interconnectedness between pro- 
gram development and outcomes assessment. Deardorff articulates the unique considerations of assessment in the context of international education and fills a gap in the extant scholarship of assessment in higher and international education. Deardorff acknowledges the book is written primarily from a US perspective, but posits that readers from outside the US may find the content useful. To that extent, the author provides a clear overview of assessment and takes care throughout the text to make effort to include international perspectives, in particular, discussing the state of assessment beyond the US and referring to host communities periodically in the text. As this book is one of the first of its kind for international education, the author does provide a good context and tools for educators outside of the US to better understand the culture of outcomes assessment. Future research designed to stimulate a deeper discussion about the expectations and impact of the culture of US outcomes assessment and accountability in international education would be valued. For example, consideration of approaches to US outcomes assessment and the resulting impact and influence on host community settings as a philosophical and values-oriented discussion would contribute to deeper understanding and dialogue in education abroad. Nonetheless, this book provides a succinct overview to international education outcomes assessment in a clear framework that all stakeholders of international education from within the US and beyond will find useful.

The book starts with an attention-grabbing introduction in Chapter One that reviews common myths of international education assessment. These myths challenge many current practices that exist in international education. Readers may recognize their own institution's assessment strategies on this list. Deardorff continues in Chapter One to introduce important framing language and terminology to ensure readers have a common framework. Chapter Two reviews 30 questions about assessment in international education. These are questions that can guide an internal review of your own institutional assessment practices, help you think through questions you may not have originally anticipated and present information in a new way for stakeholders that may be part of the assessment process within your organization.

Perhaps the most impactful and unique aspect of this book is Deardorff's introduction of the Program Logic Model Framework discussed in Chapter Five. Logic Models have long been used in international development and throughout the nonprofit sector as a mechanism to evaluate program effectiveness. The field of international education will benefit immensely from approaching assessment through a logic model framework, which provides a holistic lens for incorporating assessment into the comprehensive program. This is the only book currently in publication that 
considers how to effectively utilize a logic model in the international education context.

The text is filled with easy to utilize, concrete assessment methods that can be comfortably adopted across many institutional contexts. There are seven supporting appendices, including quizzes, self-assessments, worksheets, rubrics and institutional examples that add valuable resources to help international educators find starting points to implement the strategies presented throughout the book in practice. A wealth of information is provided to ensure international educators move beyond the haphazard methods of assessment commonly utilized today and to establish a clear framework for the field to adopt sound and successful assessment practices to measure student learning outcomes.

Beyond the innovative approach of introducing program logic models to the field of international education, the clear strength of this book is the incredible usability of the tools and strategies presented throughout the text. Whether you are involved as a graduate student or faculty in a course on assessment in higher education, working in administration of international programs and services, or simply want to learn more about assessment of student learning outcomes with consideration for the diverse learners on university campuses today, this book will provide useful information to help educators better understand the importance of assessing student learning outcomes and execute an assessment plan from start to finish.

PAIGE E. BUTLER is an Assistant Professor in the Master's Degree program of International Education Management at the Middlebury Institute of International Studies at Monterey (CA). Dr. Butler teaches international education courses focused on Student Development Theory, Education Abroad and Program Design and Assessment. Paige previously held administrative and faculty positions at CEA and Arizona State University. She holds an M.Ed and Ed.D. from Arizona State University in Educational Leadership and Policy Studies in Higher Education. Contact Dr. Butler with any comments or questions: PButler@miis.edu 